\title{
Influence of Coulomb repulsion on the Aharonov-Bohm effect in a quantum dot
}

\author{
C. W. J. Beenakker, H. van Houten, and A. A. M. Staring* \\ Philips Research Laboratories, 5600 JA Eindhoven, The Netherlands
}

(Received 25 March 1991)

\begin{abstract}
We consider the Aharonov-Bohm (AB) effect in the quantum Hall regime due to resonant tunneling into circulating edge states in a disk-shaped two-dimensional electron gas. We point out that the $A B$ effect requires the incremental charging of the disk by single electrons. The charging energy is shown to reduce the frequency of the magnetoconductance oscillations. We predict the phenomenon of the "Coulomb blockade of the Aharonov-Bohm effect": The AB effect is suppressed in a disk of small capacitance, but can be recovered by making a large hole in the center of the disk.
\end{abstract}

The effect predicted by Aharonov and Bohm ${ }^{1}$ involves the influence of the vector potential on electrons confined to a multiply connected region, within which the magnetic field is zero. The energy levels of the electrons are periodic in the enclosed flux with period $h / e$, as a consequence of gauge invariance. Electron-electron interactions do not affect this periodicity.

In the solid state, the Aharonov-Bohm (AB) effect refers to the periodic oscillations of the conductance of a ring as a function of an applied perpendicular magnetic field $B$. The periodicity of the oscillations is $\Delta B=h / e A$ in a ring enclosing an area $A$ (plus possibly harmonics, e.g., at $h / 2 e A) .^{2}$ An essential difference with the original $\mathrm{AB}$ effect is that now the field penetrates the ring itself, as well as its interior. The periodicity in the enclosed flux is therefore not exactly $h / e$. The nonzero magnetic field in the conducting region of the ring (of area $S$ ) can vary the frequency of the magnetoconductance oscillations by an amount of $(h / e S)^{-1}$. These effects are well understood in terms of the properties of a noninteracting electron gas. ${ }^{2-5}$

A remarkable consequence of the penetration of a strong magnetic field into the conducting region is that periodic magnetoconductance oscillations can occur also in a singly connected geometry, such as a point contact, ${ }^{6}$ or a disk-shaped region in a two-dimensional electron gas (a "quantum dot") ${ }^{7,8}$ The AB effect in these systems is a result of transport via edge states, which in the quantumHall-effect regime are the current-carrying states at the Fermi level. As shown by Sivan and Imry, ${ }^{9}$ edge states circulating along the boundary of a quantum dot make the geometry effectively doubly connected-in the sense that circulating edge states enclose a well-defined amount of flux.

There is, however, a difference which has not been noticed previously. In each period $\Delta B$ the number of states below a given energy increases by 1 in a quantum dotbut stays constant in a ring (with $S \ll A$ ). This is illustrated in Figs. 1(a) and 1(b), which show the energy levels as a function of $B$ for the two geometries. ${ }^{10}$ [The intermediate case $S \simeq A$ is shown as well, for comparison, in Fig. 1(c). ${ }^{10}$ ] As a result, the $A B$ magnetoconductance oscillations of a quantum dot are accompanied by an increase of the charge of the dot by one elementary charge per period. That is of no consequence if the Coulomb repulsion of the electrons can be neglected, but becomes important if the dot has a small capacitance $C$ to its surroundings, since then the electrostatic energy $e^{2} / C$ associated with the incremental charging by single electrons has to be taken into account. As we will show below, the charging energy enhances $\Delta B$ by a factor $1+e^{2} / C \Delta E$, with $\Delta E$ the separation in energy of the edge states [see Fig. 1(a)]. If $e^{2} / C \gtrsim \hbar \omega_{c}$ (with $\omega_{c} \equiv e B / m$ the cyclotron frequency) the $\mathrm{AB}$ magnetoconductance oscillations are effectively blocked by the Coulomb repulsion. We refer to this phenomenon as the Coulomb blockade of the Aharonov-Bohm effect.

To analyze the effect of the Coulomb repulsion on the periodicity of the $\mathrm{AB}$ oscillations we make use of the concepts developed in the context of the Coulomb blockade of tunneling. ${ }^{11}$ We consider the geometry of Fig. 2(a), consisting of a two-dimensional electron gas (2D EG) in which a disk-shaped region is defined electrostatically by means of a gate. This "quantum dot" is separated from the two adjacent 2D EG regions (the "reservoirs") by tunnel barriers. A current $I$ can be passed through the dot by applying a voltage difference $V$ between the two reservoirs. The conductance $G$ of the quantum dot is defined as $G=I / V$, in the limit $V \rightarrow 0$.

The probability $P(N)$ to find $N$ electrons in the quantum dot in equilibrium with the reservoirs is given by the grand canonical distribution function

$$
P(N)=\text { const } \times \exp \left(-\frac{1}{k T}\left[F(N)-N E_{F}\right]\right) .
$$

Here $F(N)$ is the free energy of the dot, $T$ the temperature, and $E_{F}$ the Fermi energy of the reservoirs. In general, $P(N)$ at $T=0$ is nonzero for a single value of $N$ only [namely, the integer which minimizes the exponent in Eq. (1)]. In that case, $G \rightarrow 0$ in the limit $T \rightarrow 0$. As discussed by Glazman and Shekhter, ${ }^{12}$ a nonzero $G$ is possible only if $P(N)$ and $P(N+1)$ are both nonzero for some $N$. Then a small applied voltage is sufficient to 
induce a current through the dot, via intermediate states $N \rightarrow N+1 \rightarrow N \rightarrow N+1 \rightarrow \cdots$. To have $P(N)$ and $P(N+1)$ both nonzero at $T=0$ requires that both $N$ and $N+1$ minimize the exponent, so that

$$
F(N+1)-(N+1) E_{F}=F(N)-N E_{F} .
$$

At $T=0$ the free energy $F(N)$ equals the ground-state energy $\mathcal{E}(N)$ of the dot. We conclude that a peak in the low-temperature conductance occurs whenever

$$
\mathcal{E}(N+1)-\mathcal{E}(N)=E_{F},
$$

for some integer $N$. Equation (3) equates the equilibrium
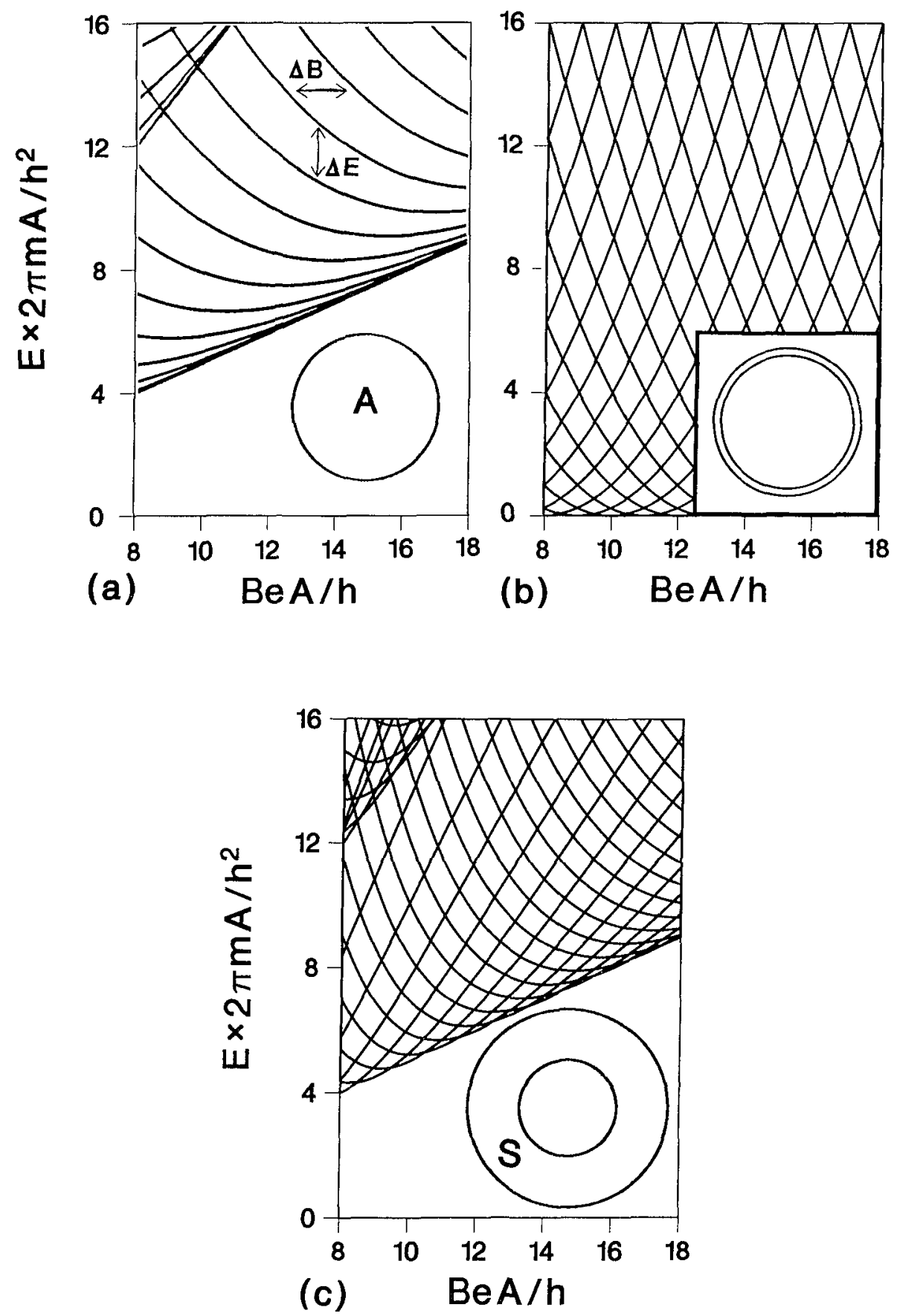

FIG. 1. Energy levels as a function of $B$ for three geometries (Ref. 10): (a) Circular disk defined by a hard-wall confining potential. Notice the asymptote corresponding to the lowest Landau level. The second Landau level is visible in the upper lefthand corner. The states between the Landau levels are the edge states. (b) Narrow circular ring of width $W \ll l_{m} \equiv(\hbar / e B)^{1 / 2}$, with the energy relative to the one-dimensional subband bottom (which is a $B$-independent constant for $W \ll l_{m}$ ). (c) Approximate energy levels in a relatively wide ring, defined by the potential $V(r)=\frac{1}{2} m \omega_{0}^{2}\left(r-r_{0}\right)^{2}$, with $A=\pi r_{0}^{2}$ and $\omega_{0} m A / h=10$. The inset shows the region (of area $S$ ) which is accessible classically by electrons in the energy range shown in the figure. 
electrochemical potentials of dot and reservoir. Since the location of the peaks in the linear-response conductance of the quantum dot is determined by its equilibrium properties, the tunneling rates between dot and reservoirs do not enter in Eq. (3) (but they do determine the amplitude of the peaks, see below).

To estimate $\mathcal{E}(N)$ we adopt the simple approximation usually made in studies of the Coulomb blockade, ${ }^{11,12}$ of taking the Coulomb interaction only into account via the macroscopic electrostatic energy $\int \phi(Q) d Q$. Here $Q=$ $-N e$ is the charge on the dot, and $\phi(Q)=Q / C+\phi_{\text {ext }}$ is the potential difference between dot and reservoir, including an external contribution $\phi_{\text {ext }}$ from the gate and from ionized donors in the heterostructure. We thus write for the ground-state energy

$$
\mathcal{E}(N)=\sum_{p=1}^{N} E_{p}+(N e)^{2} / 2 C-N e \phi_{\mathrm{ext}},
$$

where $E_{p}(p=1,2, \ldots)$ are the single-electron energy levels in ascending order, measured relative to the bottom
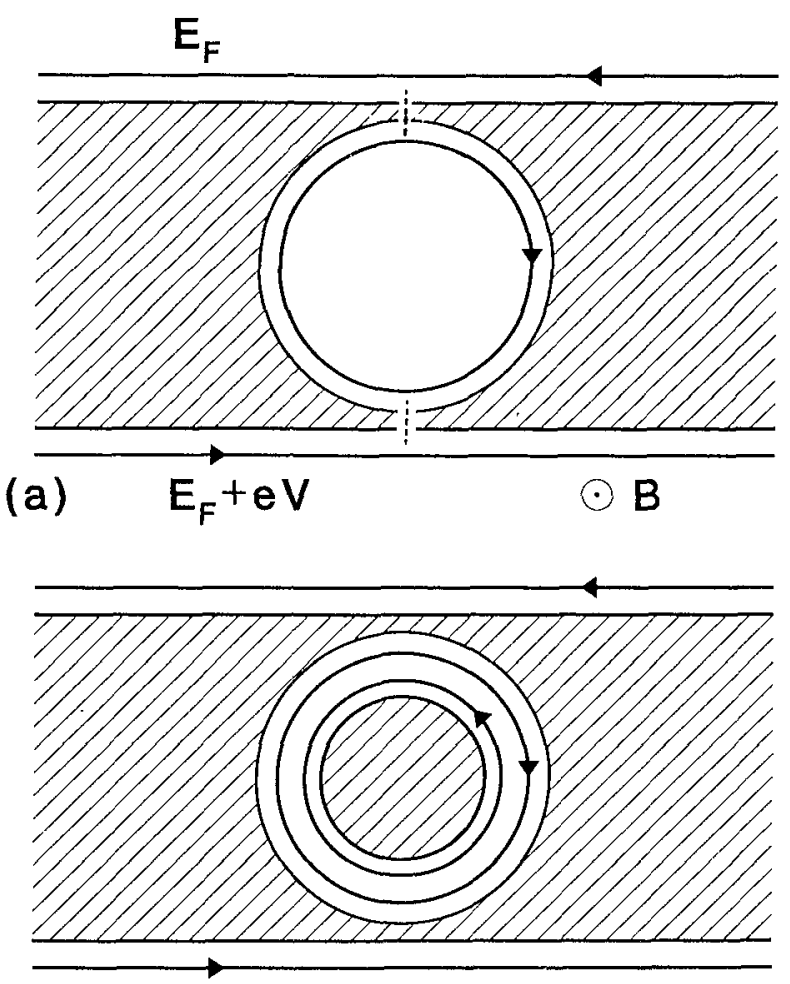

(b)

FIG. 2. (a) Quantum dot geometry. A gate (shaded) isolates a disk-shaped region in a two-dimensional electron gas from two reservoirs. Conduction through the dot occurs by tunneling (dashed lines). Edge states are indicated, with arrows pointing into the direction of motion. (b) Geometry which can be transformed from a disk into a ring. The inner perimeter of the ring supports a second set of edge states, which travel around the ring in the opposite direction. Resonant tunneling occurs predominantly via the edge states at the outer perimeter, since those at the inner perimeter have a much smaller tunneling rate. of the potential well in the quantum dot. The energy levels $E_{p}$ depend on gate voltage and magnetic field, but are assumed to be independent of $N$. This assumption is supported by self-consistent solutions of the Schrödinger and Poisson equation in a quantum dot. ${ }^{13}$

Substitution of Eq. (4) into Eq. (3) gives

$$
E_{N}^{*} \equiv E_{N}+\left(N-\frac{1}{2}\right) \frac{e^{2}}{C}=E_{F}+e \phi_{\text {ext }}
$$

as the condition for a conductance peak. The left-hand side of Eq. (5) defines a renormalized energy level $E_{N}^{*}$. The renormalized level spacing $\Delta E^{*}=\Delta E+e^{2} / C$ is enhanced above the bare level spacing by the charging energy. In the quantum limit $e^{2} / C \Delta E \rightarrow 0$, Eq. (5) is the usual condition for resonant tunneling. In the classical limit $e^{2} / C \Delta E \rightarrow \infty$, and for $B=0$, Eq. (5) describes the periodicity of the Coulomb oscillations in the conductance versus electron density (or gate voltage), studied theoretically in several papers. ${ }^{11-15}$

Equation (5) is sufficient to determine the periodicity of the conductance oscillations, but gives no information on their amplitude and width. That requires the solution of a kinetic equation, with input of the tunneling rates $\Gamma_{p}^{ \pm}$of level $p$ through the two barriers. Such a calculation has been performed by Averin and co-workers ${ }^{16}$ for the nonlinear $I-V$ characteristic of a quantum dot, in the regime $h \Gamma \ll k T$ so that the finite width of the transmission resonance can be neglected. (In this regime one has $G \ll e^{2} / h$, which is the condition under which a description of resonant tunneling in terms of a kinetic equation is valid. ${ }^{11}$ ) A similar calculation by one of us for the linear-response conductance, described elsewhere ${ }^{17}$ gives the result

$$
\begin{aligned}
& G=\frac{e^{2}}{k T} \sum_{N=0}^{\infty} \sum_{p=1}^{\infty} \frac{\Gamma_{p}^{+} \Gamma_{p}^{-}}{\Gamma_{p}^{+}+\Gamma_{p}^{-}} P(N) f\left(E_{p} \mid N\right) \\
& \times\left[1-f_{\mathrm{FD}}\left(E_{p}+\left(N-\frac{1}{2}\right) e^{2} / C\right.\right. \\
&\left.\left.-e \phi_{\mathrm{ext}}-E_{F}\right)\right],
\end{aligned}
$$

where $f_{\mathrm{FD}}(x) \equiv[1+\exp (x / k T)]^{-1}$ is the Fermi-Dirac distribution function, and $f\left(E_{p} \mid N\right)$ is the conditional probability that level $p$ is occupied given that the quantum dot contains $N$ electrons. It is worth emphasizing that this probability, as it follows from the Gibbs distribution, is different from the Fermi-Dirac distribution when $k T \simeq \Delta E$. Equation (6) agrees with the results of Ref. 12 in the classical limit $k T \gg \Delta E$ where the discreteness of the levels is unimportant. In the quantum limit $k T \ll \Delta E$ of present interest only the term $p=N=N_{\min }$ remains in Eq. (6), where $N_{\min }$ minimizes the absolute value of

$$
\Delta(N) \equiv E_{N}+\left(N-\frac{1}{2}\right) \frac{e^{2}}{C}-e \phi_{\text {ext }}-E_{F} .
$$

One then has $f\left(E_{N} \mid N\right)=1, P\left(N_{\min }\right)=f_{\mathrm{FD}}\left(\Delta\left(N_{\min }\right)\right)$, so that Eq. (6) reduces to

$$
G=-e^{2} f_{\mathrm{FD}}^{\prime}\left(\Delta\left(N_{\min }\right)\right) \frac{\Gamma_{N_{\min }}^{+} \Gamma_{N_{\min }}^{-}}{\Gamma_{N_{\min }}^{+}+\Gamma_{N_{\min }}^{-}} .
$$


Here we have used the identity $f_{\mathrm{FD}}\left(1-f_{\mathrm{FD}}\right)=-k T f_{\mathrm{FD}}^{\prime}$. The conductance peaks are centered at $\Delta\left(N_{\min }\right)=0$, which is just the condition (5) obtained above by an elementary argument.

We now apply Eq. (5) to the periodicity of the AB oscillations in a quantum dot. As discussed for a noninteracting electron gas in Refs. 7 and $8, \mathrm{AB}$ oscillations result from resonant tunneling through the quantum dot via edge states circulating along the dot perimeter [see Fig. 2(a)]. We consider here only the edge states from the lowest (spin-split) Landau level, so that the AB oscillations have a single periodicity. This corresponds to the strong-magnetic-field limit. The function $E_{p}(B)$ can be approximated by a sequence of equidistant parallel lines,

$$
E_{p}=\text { const }-\frac{\Delta E}{\Delta B}(B-p \Delta B)
$$

over a limited field range. Equation (8) holds only for a few periods $\Delta B$ in the field range shown in Fig. 1(a); a much larger field range of nearly equidistant edge states is possible at higher $B$, as is shown for example in Fig. 2 of Ref. 8. Sivan and Imry estimate ${ }^{9} \Delta B \approx h / e A$ and $\Delta E \approx \hbar \omega_{c} l_{m} / 2 R$, where $l_{m} \equiv(\hbar / e B)^{1 / 2}$ is the magnetic length and $R \gg l_{m}$ is the radius of the (circular) quantum dot. These are order-of-magnitude estimates for a hardwall confining potential. ${ }^{18} \mathrm{On}$ substitution of $\mathrm{Eq}$. (8) into Eq. (5), one finds that the magnetic-field value $B_{N}$ of the $N$ th conduction peak is determined by

$$
N\left(\Delta E+\frac{e^{2}}{C}\right)=\frac{\Delta E}{\Delta B} B_{N}+E_{F}+\text { const. }
$$

The $B$ dependence of the reservoir Fermi energy can be neglected in Eq. (9) in the case of a hard-wall confining potential (since $d E_{F} / d B \approx \hbar \omega_{c} / B \ll \Delta E / \Delta B$ ). The period $\Delta B^{*}$ of the $A B$ magnetoconductance oscillations is then given by

$$
\Delta B^{*} \equiv B_{N+1}-B_{N}=\Delta B\left(1+\frac{e^{2}}{C \Delta E}\right) .
$$

This result implies that the charging energy enhances the spacing of two subsequent peaks in $G$ versus $B$ by a factor $1+e^{2} / C \Delta E$. The periodicity of the magnetoconductance oscillations is lost if $\Delta B^{*}$ becomes so large that the approximation (8) for $E_{p}(B)$ (with a $B$-independent spacing $\Delta B$ ) breaks down. Since Eq. (8) holds at most over an energy range of the Landau-level separation $\hbar \omega_{c}$, this Coulomb blockade of the $\mathrm{AB}$ effect (i.e., the disappearance due to the charging energy of magnetoconductance oscillations with a constant periodicity) occurs when $(\Delta E / \Delta B) \Delta B^{*} \gtrsim \hbar \omega_{c}$, i.e., when $e^{2} / C \gtrsim \hbar \omega_{c}$.

The AB oscillations with bare periodicity $\Delta B=h / e A$ are recovered if one makes a large hole in the disk. In contrast to a disk, a ring supports both clockwise and counterclockwise propagation. The two sets of states which circulate in opposite directions along the inner and outer perimeter are distinguished by the opposite sign of $d E_{p} / d B$, i.e., of the magnetic moment. A disk has only an outer perimeter, and supports edge states circulating in one direction only. These states consequently all have the same sign of $d E_{p} / d B$.
Consider first the case of a very narrow ring, of width $W \ll l_{m}$. The energy levels for this case are shown in Fig. 1(b). ${ }^{10}$ The states $E_{p}(B)$ all lie on the set of translated parabolas $E_{q}(B)=\left(h^{2} / 8 \pi m A\right)(q-B / \Delta B)$, with $\Delta B=h / e A$ and $q$ a positive or negative integer. The set of levels $\left\{E_{p}(B)\right\}$ is obtained by ordering the set $\left\{E_{q}(B)\right\}$ in ascending order. Since the two sets $\left\{E_{q}(B)\right\}$ and $\left\{E_{\mathrm{q}}(B+\Delta B)\right\}$ are identical, it follows that

$$
E_{p}(B)=E_{p}(B+\Delta B),
$$

which guarantees, in combination with Eq. (5), that the $A B$ periodicity equals $\Delta B$ regardless of the charging energy. In a disk, in contrast, one has according to Eq. (8),

$$
E_{p}(B)=E_{p+1}(B+\Delta B)
$$

which in combination with Eq. (5) yields a periodicity $\Delta B^{*}$ enhanced above $\Delta B$ by the charging energy.

To illustrate the difference, we compare in Fig. 3 for disk and narrow ring the renormalized energy levels $E_{p}^{*}$ [defined in Eq. (5)]. The effect of the charging energy in

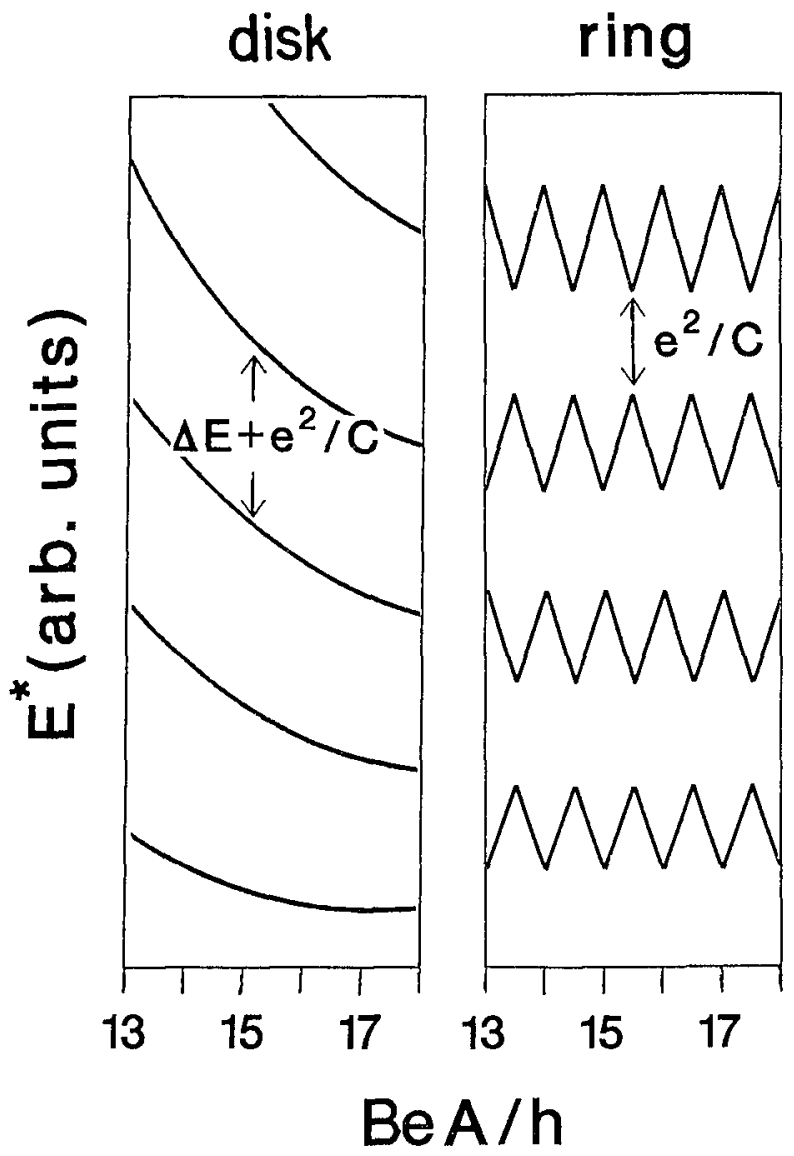

FIG. 3. Renormalized energy levels, defined by Eq. (5), corresponding to the bare energy levels in Figs. 1(a) and 1(b), for a particular (arbitrary) value of the charging energy $e^{2} / C$. Left panel, disk geometry; right panel, narrow ring geometry (the cusps will be rounded by a small amount of disorder). 
a ring (right panel) is to open an energy gap of magni tude $e^{2} / C$ in $E_{p}^{*}$ This gap will affect the periodicity of the conductance oscillations as a function of $E_{F}$, but not as a function of $B$ In a disk (left panel), the characterIstic "sawtooth" of the ring is not present in the strongmagnetic-field limit of a single Landau level ${ }^{19}$ The charging energy incieases the energy separation $\Delta E^{*}$ as well as the magnetic-field separation $\Delta B^{*}$, and thus affects the conductance oscillations in a disk both as a function of $E_{F}$ and $B$

To complete our discussion of the two himiting cases of disk and narrow ring, we now consider the intermediate case of a wide ring, with $W \gg l_{m}$ In a wide ring, Eq (11) holds only up to teims of order $S / A$ (where $S$ is the area of the conducting region, and $A$ is the enclosed area) If $S \ll A$, then $\mathrm{Eq}(11)$ is a good approximation because a field inciement $\triangle B=h / e A$ does not change the Landaulevel degeneracy $B e S / h$ (smce $\Delta B e S / h=S / A \ll 1$ ) If $S \simeq A, \mathrm{Eq}$ (11) holds for a relatively small number of periods only, as is shown $\mathrm{m} \mathrm{F}_{\mathrm{ig}}$ 1(c) In this intermediate case one would observe series of $A B$ oscillations with spacing $\Delta B$ separated by the larger spacing $\Delta B^{*}$

It is of interest to see explicitly how $\mathrm{Eq}$ (11) follows from the condition $S \ll A$ in a ring which is much wider than the magnetic length We use the fact that the edge state spectrum $\left\{E_{p}\right\}$ for $W \gg l_{m}$ is composed fiom the levels $E_{q}^{ \pm}$of edge states at the inner $(+)$ and outer (-) permeter of the ring These two sets of levels can, analogously to $\mathrm{Eq}(8)$, be described by $E_{q}^{ \pm}=$const $\pm\left(\Delta E^{ \pm} / \Delta B^{ \pm}\right)\left(B \pm q \Delta B^{ \pm}\right) \quad$ Edge states at the two perimeters have opposite sign of $d E / d B$ We now use $\Delta B^{+}=\Delta B^{-}+O(S / A)$ In the limit $S / A \rightarrow 0$, the full spectrum $\left\{E_{p}\right\}$ (obtamed by combining $\left\{E_{q}^{+}\right\}$ and $\left\{E_{q}^{-}\right\}$in ascending order) satisfies $\mathrm{Eq}$ (11), with $\Delta B=\Delta B^{ \pm}$Note that this holds regardless of the relative magnitudes of $\Delta E^{+}$and $\Delta E^{-}$
A controlled experimental demonstration of the influence of Coulomb repulsion on the $\mathrm{AB}$ effect may be obtained in a system which can be transformed from a disk into a ring What we have in mind is a geometry such as shown in Fig 2(b), whıch has an additıonal disk-shaped gate withın the gate of $\mathrm{Fig} 2$ (a) By applying a negatıve voltage to this additional gate one depletes the central region of the quantum dot, thereby transforming it into a ring In order to estimate the mutual capacitance $C$ between the undepleted quantum dot and the adjacent 2D EG reservorrs, we note that only a circular strip of width $l_{m}$ and radius $R$ along the circumference of the dot contributes to $C$ The central region of the dot is incompressible in the quantum-Hall-effect regime, and thus behaves as a dielectric as far as the electrostatics is concerned ${ }^{20}$ The capacitance $C$ contains contributions from the selfcapacitance of this stip as well as fiom its capacitance to the gate (We assume that the gate is electrically connected to the 2D LG ieservoris ) Both contilbutions are of ordel $\epsilon R$, with a numeical piefactor of order unity which depends only logarithmically on the width of the strip and the separation to the gate $\mathrm{e}^{21}(\epsilon$ is the dielectric constant) A dot radius of $1 \mu \mathrm{m}$ yields a charging energy $e^{2} / C \sim 1 \mathrm{meV}$ for $\epsilon \sim 10 \epsilon_{0}$ This exceeds the level separation $\Delta E \sim \hbar \omega_{c} l_{m} / 2 R \sim 10^{-2} \mathrm{meV}(\mathrm{T} / B)$ at a field of a few $T$ A significant increase of the frequency of the $A B$ oscillations should thus be observable on depletion of the central region of the dot, even for a relatively large radius of $1 \mu \mathrm{m}{ }^{22}$ An ultimate test of the theory presented in this paper would be to observe the Coulomb blockade of the Aharonov-Bohm effect in a submicrometer disk with $e^{2} / C \gtrsim \hbar \omega_{c}$, and the recovery of the $\mathrm{AB}$ effect on transformation to a ring

We acknowledge the stimulating support of $M F H$ Schuurmans
*Also at Eindhoven University of Technology, 5600 MB Eindhoven, The Netherlands

${ }^{1} Y$ Aharonov and D Bohm, Phys Rev 115, 485 (1959)

${ }^{2} \mathrm{~S}$ Washburn and R A Webb, Adv Phys 35, 375 (1986), A G Aionov and Yu V Sharvin, Rev Mod Phys 59,755 (1987) The $h / 2 e$ periodicity is negligible in the quantum Hall-effect regime, where the $\mathrm{AB}$ effect occurs due to resonant tunneling into circulating edge states

${ }^{3}$ J K Jain, Phys Rev Lett 60, 2074 (1988)

${ }^{4} \mathrm{G}$ Timp, $\mathrm{P} \mathrm{M}$ Mankıewich, $\mathrm{P}$ DeVegvar, R Behrınger, $\mathrm{J}$ E Cunnugham, $\mathrm{R}$ E Howard, $\mathrm{H}$ U Baranger, and $\mathrm{J}$ K Jain, Phys Rev B 39, 6227 (1989), C J B Ford, T $\mathrm{J}$ Thornton, $\mathrm{R}$ Newbury, $\mathrm{M}$ Pepper, $\mathrm{H}$ Ahmed, D C Peacock, D A Ritchie, J E $\Gamma$ Frost, and G A C Jones, Appl Phys Lett 54, 21 (1989)

${ }^{5}$ For a leview, see $\mathrm{C} W \mathrm{~J}$ Beenakker and $\mathrm{H}$ van Houten, in Solıd State Physacs, edited by H Ehrenreich and D Turnbull (Academic, New York, 1991), Vol 44, p 1

${ }^{6} \mathrm{P} H \mathrm{M}$ van Loosdrecht, $\mathrm{C}$ W $\mathrm{J}$ Beenakker, $\mathrm{H}$ van Houten, J G Willamson, B J van Wees, J E Moolj, C T Foxon, and J J Harrus, Phys Rev B 38, 10162 (1988)

${ }^{7} \mathrm{~B} \quad \mathrm{~J}$ van Wees, L $\mathrm{P}$ Kouwenhoven, C J P M Harmans,
J G Williamson, $\mathrm{C}$ C Timmering, $M$ E I Broekaart, C T Foxon, and $J$ J Harrs, Phys Rev Lett 62, 2523 (1989)

${ }^{8} \mathrm{U}$ Sivan, $\mathrm{Y}$ Imı and $\mathrm{C}$ Hartzstein, Phys Rev B 39, 1242 (1989)

${ }^{9} \mathrm{U}$ Siran and $\mathrm{Y}$ Imry, Phys Rev Lett 61, 1001 (1988) The peliodic oscillations in the magnetization of a quantum dot studied by Sivan and Imiy occur at a constant number of elections in the dot, and are therefore not affected by the charging effects discussed in the present paper

${ }^{10}$ I he energy levels for the dot are due to $\Gamma$ Geennchx, $\Gamma \mathrm{M}$ Peeters, and J T Denreese, J Appl Phys 68, 3435 (1990) The energy levels for the nanow rmg are due to $\mathrm{M}$ Buttrher, Y Imiy, and R Landaueı, Phys Lett A 96, 365 (1983) The energy levels for the wide ing follow from the BohrSommeifeld quantization iule in the approximation that the confining potential is smooth on the scale of $l_{m}$ See $C W$ $J$ Beenakker, $H$ van Houten, and A A $M$ Stanng, in Granular Nanoelectroncs, NATO Advanced Study Instrtute, Series $B$ Physics, edited by D K Ferry, J Barker, and C Jacobonı (Plenum, New Yolk in piess)

${ }^{11}$ For reviews of the field of single-election tunneling in met- 
als, we refer to K. K. Likharev, IBM J. Res. Dev. 32, 144 (1988); D. V. Averin and K. K. Likharev, in Mesoscopic Phenomena in Solids, edited by B. L. Al'tshuler, P. A. Lee, and R. A. Webb (Elsevier, Amsterdam, 1991); a recent review of single-electron tunneling in semiconductors is $H$. van Houten, C. W. J. Beenakker, and A. A. M. Staring, in Single Charge Tunneling, NATO Advanced Study Institute, Series B: Physics, edited by H. Grabert and M. H. Devoret (Plenum, New York, in press).

${ }^{12}$ L. I. Glazman and R. I. Shekhter, J. Phys. Condens. Matter 1, 5811 (1989); see also I. O. Kulik and R. I. Shekhter, Zh. Eksp. Teor. Fiz. 68, 623 (1975) [Sov. Phys.-JETP 41, 308 (1975)].

${ }^{13}$ A. Kumar, S. E. Laux, and F. Stern, Phys. Rev. B 42, 5166 (1990).

${ }^{14}$ M. Amman, K. Mullen, and E. Ben-Jacob, J. Appl. Phys. 65,339 (1989).

${ }^{15} \mathrm{H}$. van Houten and C. W. J. Beenakker, Phys. Rev. Lett. 63, 1893 (1989).

${ }^{16}$ D. V. Averin and A. N. Korotkov, Zh. Eksp. Teor. Fiz. 97, 1661 (1990) [Sov. Phys.-JETP 70, 937 (1990)]; A. N. Korotkov, D. V. Averin, and K. K. Likharev, Physica B $165 \& 166,927$ (1990).

${ }^{17}$ C. W. J. Beenakker, Phys. Rev. B 44, 1646 (1991). Equation (6) has recently been derived independently by Y. Meir, N. S. Wingreen, and P. A. Lee (unpublished).

${ }^{18}$ For a "soft" confining potential $V(r)$ (with $l_{m} V^{\prime} \lesssim \hbar \omega_{c}$ ) one has instead the estimates $\Delta B=(h / e)\left[A(B)+B A^{\prime}(B)\right]^{-1}=$ $(h / e A)\left[1-\hbar \omega_{c} / R V^{\prime}(R)\right]^{-1}$ (Ref. 7$)$, and $\Delta E=h / \tau=$ $l_{m}^{2} V^{\prime}(R) / R$, where $A(B)$ is the area enclosed by the equipotential of radius $R$ at the guiding center energy $V(R)=$
$E-\frac{1}{2} \hbar \omega_{c}$. The estimate for $\Delta E$ results from the correspondence between the level spacing and the period $\tau$ of the classical motion along the equipotential, with guidingcenter-drift velocity $V^{\prime}(R) / e B$.

${ }^{19}$ The renormalized energy levels of a disk with two occupied Landau levels do in fact have a sawtooth shape, reminiscent of the ring. In Fig. 1(a) the two-Landau-level region is visible in the upper left-hand corner. The sawtooth originates from the presence of states with positive as well as negative $d E_{p} / d B$. The circumstance that a second occupied Landau level can remove the Coulomb blockade of the AharonovBohm effect has recently been utilized experimentally by $\mathrm{P}$. L. McEuen, E. B. Foxman, U. Meirav, M. A. Kastner, Y. Meir, N. S. Wingreen, and S. J. Wind, Phys. Rev. Lett. 66, 1926 (1991).

${ }^{20}$ We thank Dr. C. Glattli for pointing this out to us.

${ }^{21}$ L. D. Landau and E. M. Lifshitz, Electrodynamics of Continuous Media (Pergamon, Oxford, 1960).

${ }^{22}$ Van Wees et al. (Ref. 7) observed AB magnetoconductance oscillations in the quantum-Hall-effect regime in a dot with a radius of $0.75 \mu \mathrm{m}$. According to the estimates given in the text, the charging energy should have a significant effect on the periodicity of the AB oscillations in this system, in the case of a single Landau level in the dot. The data reported in Ref. 7 were, however, all taken under conditions that several (spin-split) Landau levels were occupied in the dot, some of which extended over the potential barriers into the reservoirs. Since the charge on the dot can then vary continuously, the Coulomb blockade of the $A B$ effect is inoperative. 